(Aus der Abteilung für Hautkrankheiten der Budapester Graf Albert ApponyiPoliklinik [Vorstand: Prof. Ludwig Török].)

\title{
Über einen Fall von Granuloma annulare.
}

\author{
Von \\ Dr. Desider Kenedy, \\ Assistent.
}

Mit 1 Textabbildung.

(Eingegangen am 25. Dezember 1921.)

Seit der Veröffentlichung der Arbeit Colcott Fox's, der das Granuloma annulare im Jahre 1895 zuerst beschrieb, erschienen mehrere Mitteilungen über diese Affektion. Die Krankheit wurde von den verschiedenen Autoren mit verschiedenen Namen belegt, die teilweise bloß auf den klinisch-morphologischen Charakter der Hautveränderungen Bezug hatten (Colcott Fox, Ringed eruption of the fingers; Galloway, Lichen annularis; D ubreuilh, Eruption circinée chronique de la main usw.), teilweise aber durch die Auffassung der Autoren von der Natur der pathologisch-anatomischen Veränderung der Haut bedingt waren (Rasch und Gregerson, Neuer Typus benigner sarkoider Geschwülste der Haut; Broeq, Néoplasie nodulaire et circinée des extrémités; Audry, Erythémato-sclérose circinée du dos de la main usw.). Im folgenden teile ich einen Fall mit, den wir Gelegenheit hatten im vorigen Jahre an unserer Abteilung zu beobachten.

R. J., 60 Jahre alte Kaufmannsfrau. Eltern im hohem Alter gestorben, zwei Geschwister leben und sind gesund. Thr Mann starb an Herzleiden. Vier Kinder sind im Alter von 2, 9, 10 und 22 Jahren gestorben. Pat. leidet seit 10 Jahren an Diabetes, hat früher außerdem keine erwähnenswerten Erkrankungen durchgemacht. Sie suchte wegen ihrer Zuckerkrankheit jedes Jahr Karlsbad auf, der Urin blieb aber nur für kurze Zeit zuckerfrei.

Thr Hautleiden begann von 4 Jahren am rechten Handrücken. Pat. gibt an, daß die ersten Erscheinungen sich nach einem Mückenstiche zeigten. Die damals entstandenen rote Flecke blieben einige Monate bestehen, obne subjektive Beschwerden zu verursachen, und verschwanden nachher spurlos. Über den weiteren Verlauf der Krankheit sind die Angaben der Pat. lückenhaft und unsicher. So viel läßt sich aus ihren Angaben feststellen, da $ß$ nach Abheilung der Flecke sich sukzessive an anderen Stellen neue zeigten, zuerst am rechten, dann am linken Handrücken. Dieselben waren zu Beginn linsengroß, wuchsen allmählich bis zur Größe eines 20-Hellerstückes, während sie gleichzeitig in der Mitte abheilten, und nur an ihrem Rande verblieb ein schmaler Saum. Auf diese Weise entstanden ringförmige Herde 
und nach Verschwinden eines Teiles des Saumes bogenförmige. Nach mehrmonatigem Bestehen verschwand einer oder der andere der Flecke, ohne Narben zu hinterlassen. Der jüngst entstandene Herd besteht beiläufig seit dem August 1920. Von Jucken oder Schmerzen waren die Erscheinungen nie begleitet.

Status praesens 1. XI. 1920: Mittelhohe, gut genährte Pat. Innere Organe ohne Befund. In der Lunge ist auch röntgenologisch keine Veränderung nachzuweisen. Schleimhäute sind frei. Wassermannsche Reaktion negativ. Blutbild normal. Pirquet, Ponndorf negativ. Urinbefund: Eiweiß in Spuren, Zucker 4,9\%, Aceton in Spuren. Im Sediment einige Eiter- und Epithelzellen.

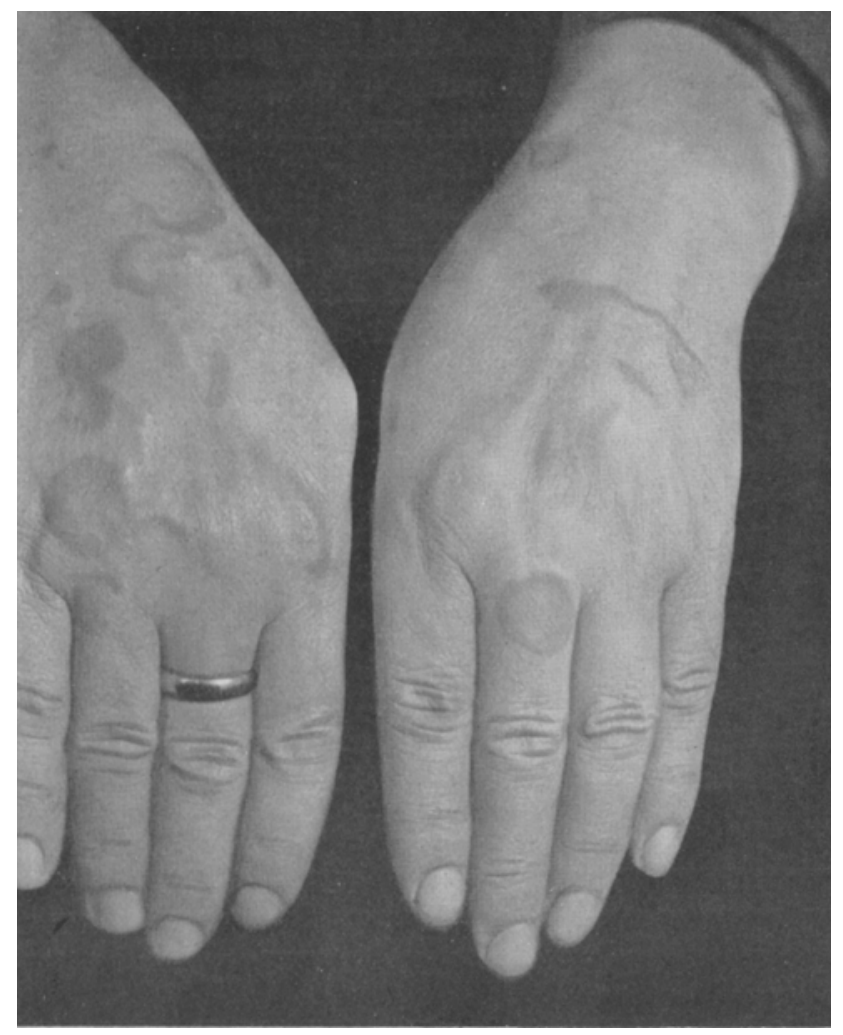

Hautstatus: Die Hautveränderungen sind an beiden Handrücken, über beiden Metakarpalgelenken und am 3. Glied des mittleren Fingers der linken Hand (s. Abbildung) lokalisiert. Sie bilden teils linsen- bis 1-Kronenstück große, geschlossene ringförmige, teils bogenförmige oder girlandenartige Herde. Am linken Handrücken ist auch ein Herd in Form einer querwellig verlaufenden Linie zu sehen. Die jüungeren Herde zeigen im zentralen Teile eine leichte Einsenkung, bei den älteren und größeren Herden ist im Zentrum eine auf das Normale zurückgesunkene, etwas pigmentierte, sonst normale Haut zu sehen. Die Randpartie der Veränderung wird durch einen 2--21/2 $\mathrm{mm}$ breiten wallartigen Saum gebildet, der sich gegen die Peripherie steil über die normale Haut erhebt, zentral allmählich abfällt. Die kleinen 
Herde zeigen eine bräunlichrote Farbe, der erhabene Saum der gröleren Herde ist blasser, hauptsächlich auswärts, wo er bei geballter Faust anämisch, elfenbeinartig aussieht. Dieses elfenbeinartige Aussehen läßt sich durch einen leichten Druck, der durch geringe Spannung der Haut ausgeübt wird, deutlich hervorrufen. An der Stelle des Überganges von der Anämie des Randwalles zur zentralwärts gelegenen Hyperämie sind kleine Teleangiektasien wahrzunehmen. Hier ist die bräunlichrote Färbung deutlich; diese geht in einem ganz schmalen Streifen von beiläufig $1 / 2-1 \mathrm{~mm}$ auch auf die, in normalem Niveau liegende Haut über. Im Epithel ist keine Veränderung vorhanden. Die Hautfelderung ist deutlich sichtbar. Dem Randwalle entsprechend läßt sich in der ganzen Dicke des Coriums eine derbe Infiltration tasten. Die regionären Drüsen zeigen keine Veränderung.

Von einem frischen kleinen und von einem größeren, länger bestehenden Herd excidiertes Material wurde in Formalin fixiert, in Paraffin eingebettet und in Serienschnitten verarbeitet. Die histologische Untersuchung ergab folgendes:

Im Epithel sind keine krankhaften Veränderungen vorhanden. Im Corium ist schon bei kleiner Vergrößerung ein Reichtum an Zellkernen wahrzunehmen, der sich auf die ganze Dicke desselben erstreckt. Die Kerne sind nicht gleichmäßig angeordnet, sondern reihen sich in gestreckten oder netzförmig verbundenen Zügen und in kleinen Häufchen aneinander. Im Stratum reticulare erreicht die Zellenvermehrung einen höheren Grad als im Stratum papillare. Einzelne Züge ziehen fast bis zur Corioepithelgrenze hinauf. Das kollagene Gewebe ist nicht vermehrt. In der Umgebung der Zellhaufen und Züge ist es in mit Eosin gefärbten Präparaten etwas heller gefärbt, leuchtend, die einzelnen Bündel sind voluminöser, etwas homogenisiert. In den nach van Gieson gefärbten Präparaten zeigen sich hier ganz schmale Streifen von gelbgefärbtem Bindegewebe. Die Gelbfärbung erstreckt sich kaum auf einige kollagene Fasern. Bei stärkerer Vergrößerung zeigen sich die vermehrten Zellen als zum Typus der Fibroblasten oder Epitheloidzellen gehörend. Ihr Körper ist gut wahrzunehmen, die Kerne oval, spindel- oder sternförmig. In den Zügen und Häufchen finden wir stellenweise Kern an Kern, von denen sich manche schwächer färben, kleine Ausläufer haben, durch welche sie miteinander in Verbindung zu stehen scheinen.

Entzündungserscheinungen waren nicht nachzuweisen. Plasmazellen fanden wir nicht, Riesenzellen auch nicht. Die elastischen Fasern waren erhalten, nur im Gebiete der Zellhaufen etwas verdrängt. Mit Färbung nach ZiehI-Neelsen konnten wir keine Bacillen nachweisen.

Die Patientin wurde zuerst mit Arsen behandelt, welches sie aber nicht vertrug; wir unterzogen sie hierauf einer Röntgenbehandlung. Wir bestrahlten zuerst bloß die linke Hand und nach vollständiger Heilung derselben die rechte. Auf drei, unter der Erythemdosis bleibende, in Intervallen von 4 Wochen vorgenommene Bestrahlungen waren sämtliche Herde verschwunden, die Hand, mit Ausnahme einer geringen 
Pigmentation, von vollkommen normalem Aussehen. Narben oder Atrophie, auch die geringste Andeutung derselben, waren nicht nachzuweisen.

Bei der ersten Untersuchung der Patientin - wir hatten bisher noch keinen Fall von Granuloma annulare an unserer Abteilung zu sehen Gelegenheit gehabt - stellten wir die Diagnose, den klinisch feststellbaren pathoanatomischen Veränderungen und dem klinischen Verlauf gemäß, auf ein Granulom. Bei der Differentialdiagnose kam neben dem Granuloma annulare Lues in Frage, und so leiteten wir trotz der negativen Wassermannschen Reaktion bis zur Untersuchung des excidierten Materials eine antiluetische Kur ein. Diese Therapie blieb erfolglos. Die Diagnose Lues ließen wir demnach fallen.

Die klinische Diagnose Granuloma annulare wurde durch den histologischen Befund insoferne nicht unterstützt, als wir bloß eine Hyperplasie, eine Gewebsneubildung der Bindegewebszellen fanden. Regressive Erscheinungen waren höchstens leicht angedeutet. [Geringe Degenerationssymptome der Zellkerne (die durch die schmalen Ausläufer der Zellkerne und ihre stelleneise schwächere Färbung angedeutet wurden. Beginnende Karyolyse?) und des Bindegewebes das sich stellenweise ebenfalls etwas schlechter färbt und wie homogenisiert erscheint. (Beginnende hyaline Degeneration?)] Entzündliche Veränderungen waren überhaupt nicht zugegen.

Wir wandten uns brieflich an Herrn Professor Arndt, der in einer zusammenfassenden Arbeit (dieses Archiv 108. 1911) über das Granuloma annulare ausführlich berichtet hat und bezüglich der Widersprüche der histologischen Befunde der verschiedenen Untersucher meint, daß sie sich zum Teile daraus erklären lassen, daß verschiedene Entwicklungsstadien untersucht und nicht immer Serienschnitte angefertigt worden sind. In seiner Antwort schreibt Arndt, dem wir die Photographie nebst der Krankengeschichte und ein histologisches Präparat des Falles eingesendet hatten: „Was Thren Fall anbelangt, so würde ich denselben als Granuloma annulare auffassen. Das klinische Bild erscheint mir so charakteristisch, daß gewisse Abweichungen des histologischen Bildes von den klassischen Fällen (Fehlen der eigenartigen Bindegewebsnekrose) nicht genügen, um die Diagnose Granuloma annulare abzulehnen..."

Vom klinisch-diagnostischen Standpunkt steht das Granuloma annulare entschieden der Gruppe der Granulome am nächsten. So wie bei den übrigen in diese Gruppe gehörigen Krankheiten wird auch das Granuloma annulare durch den chronischen Verlauf eines Zelleninfiltrates im Bindegewebe der Haut, durch das periphere Fortschreiten der einzelnen Herde, durch die Fähigkeit derselben zur spontanen Rückbildung charakterisiert. Mangel an makroskopisch nachweisbaren regressiven Erscheinungen finden wir manchmal auch bei anderen Granulomen. 
Das vollständige Fehlen von entzündlichen Veränderungen bildet dagegen einen wesentlichen Unterschied zwischen unserem Fall und den Granulomen. Auch die Hyperämie war in unserem Fall keine entzündliche, sondern durch Teleangiektasien und Blutstauung bedingt. Die Hyperplasie der Bindegewebszellen, der Mangel an entzündlichen Erscheinungen veranlassen uns, den Proze $B$ - uns der Meinung Rasch und Gregersons (s. dieses Archiv 64) anschließend -- eher den Geschwülsten der Bindegewebsgruppe nahezustellen. Seiner Struktur und seinem Verlaufe nach scheint es dem Kaposischen idiopathischen Sarkom am nächsten zu stehen. Aber auch von diesem unterscheidet es sich durch die größere Neigung zu spontaner Rückbildung, den Mangel von größeren und diffusen Zellhyperplasien, den geringeren Umfang der letztere zusammensetzenden Zellen, den Mangel an Hämorrhagien und Pigmentation, seine geringere Ausbreitung und Benignität.

Zum Schlusse wollen wir noch erwähnen, daß ebenso wie in den Tabellen von Della Favera nnd Klausner (dieses Archiv 120. 1914) auch in unserem Falle Diabetes vorhanden war.

Anmerkung bei der Korrektur. Wir haben die Patientin am 12. II. 1922 wiedergesehen. Die Hauterscheinungen waren (die jüngsten mit Hinterlassung Jeichter Pigmentation) vergangen. Zuckergehalt des Urines vor 6 Wochen $5 \%$, am 11. II. $4,8 \%$. 